\title{
The cinnamyl alcohol dehydrogenase gene family in Populus: phylogeny, organization, and expression
} \author{
Abdelali Barakat*1, Agnieszka Bagniewska-Zadworna², Alex Choi ${ }^{3}$, \\ Urmila Plakkat ${ }^{1}$, Denis S DiLoreto ${ }^{1}$, Priyadarshini Yellanki ${ }^{1}$ and \\ John E Carlson*4
}

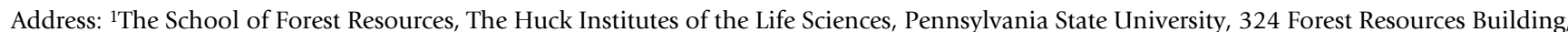
University Park, PA 16802, USA, ${ }^{2}$ Department of General Botany, Institute of Experimental Biology, Adam Mickiewicz University, Umultowska 89, 61-614 Poznań, Poland, 3Schreyer Honors College, Pennsylvania State University, 10 Schreyer Honors College, University Park, PA 16802, USA and ${ }^{4}$ The School of Forest Resources, Department of Horticulture, The Huck Institutes of the Life Sciences, Pennsylvania State University, 323 Forest Resources Building, University Park, PA 16802, USA

Email: Abdelali Barakat* - aub14@psu.edu; Agnieszka Bagniewska-Zadworna - agabag@amu.edu.pl; Alex Choi - ayc5056@psu.edu; Urmila Plakkat - uxp107@psu.edu; Denis S DiLoreto - dsd134@psu.edu; Priyadarshini Yellanki - ylpd@yahoo.com;

John E Carlson* - jec16@psu.edu

* Corresponding authors
\end{abstract}

Published: 6 March 2009

BMC Plant Biology 2009, 9:26 doi:10.1 186/1471-2229-9-26
Received: 3 October 2008

Accepted: 6 March 2009

This article is available from: http://www.biomedcentral.com/147/-2229/9/26

(c) 2009 Barakat et al; licensee BioMed Central Ltd.

This is an Open Access article distributed under the terms of the Creative Commons Attribution License (http://creativecommons.org/licenses/by/2.0), which permits unrestricted use, distribution, and reproduction in any medium, provided the original work is properly cited.

\begin{abstract}
Background: Lignin is a phenolic heteropolymer in secondary cell walls that plays a major role in the development of plants and their defense against pathogens. The biosynthesis of monolignols, which represent the main component of lignin involves many enzymes. The cinnamyl alcohol dehydrogenase (CAD) is a key enzyme in lignin biosynthesis as it catalyzes the final step in the synthesis of monolignols. The CAD gene family has been studied in Arabidopsis thaliana, Oryza sativa and partially in Populus. This is the first comprehensive study on the CAD gene family in woody plants including genome organization, gene structure, phylogeny across land plant lineages, and expression profiling in Populus.
\end{abstract}

Results: The phylogenetic analyses showed that CAD genes fall into three main classes (clades), one of which is represented by CAD sequences from gymnosperms and angiosperms. The other two clades are represented by sequences only from angiosperms. All Populus CAD genes, except PoptrCAD 4 are distributed in Class II and Class III. CAD genes associated with xylem development (PoptrCAD 4 and PoptrCAD 10) belong to Class I and Class II. Most of the CAD genes are physically distributed on duplicated blocks and are still in conserved locations on the homeologous duplicated blocks. Promoter analysis of CAD genes revealed several motifs involved in gene expression modulation under various biological and physiological processes. The CAD genes showed different expression patterns in poplar with only two genes preferentially expressed in xylem tissues during lignin biosynthesis.

Conclusion: The phylogeny of CAD genes suggests that the radiation of this gene family may have occurred in the early ancestry of angiosperms. Gene distribution on the chromosomes of Populus showed that both large scale and tandem duplications contributed significantly to the CAD gene family expansion. The duplication of several CAD genes seems to be associated with a genome duplication event that happened in the ancestor of Salicaceae. Phylogenetic analyses associated with 


\begin{abstract}
expression profiling and results from previous studies suggest that CAD genes involved in wood development belong to Class I and Class II. The other CAD genes from Class II and Class III may function in plant tissues under biotic stresses. The conservation of most duplicated CAD genes, the differential distribution of motifs in their promoter regions, and the divergence of their expression profiles in various tissues of Populus plants indicate that genes in the CAD family have evolved tissue-specialized expression profiles and may have divergent functions.
\end{abstract}

\section{Background}

Lignin is a phenolic heteropolymer that provides plant cells with structural rigidity, a barrier against insects and other pestilent species, and hydrophobicity [1-4]. Its role in hydrophobicity helps xylem cells facilitate the conduction of water and minerals throughout the plant [5]. Lignin is the second most abundant plant molecule on earth next to cellulose and comprises approximately 35\% of the dry matter of wood in some tree species [6]. The composition of lignin consists of various phenylpropanoids, predominantly the monolignols p-coumaryl, coniferyl, and sinapyl alcohols. Lignin varies in content and composition between gymnosperms and angiosperms. In gymnosperms, lignin contains guaiacyl subunits ( $\mathrm{G}$ units) and $p$-hydroxyphenyl units ( $\mathrm{H}$ units) polymerized from coniferyl alcohol and from $p$-coumaryl alcohol respectively. Lignin in angiosperms comprises, in addition to Gunits and some H-units [7], syringyl units (or S-units) polymerized from sinapyl alcohol. However, there are exceptions found within each group [7] and variation in lignin composition can even occur between cell types within the same plant.

The monolignol biosynthetic pathway involves many intermediates and enzymes [8]. The first step in the process consists of a deamination of phenylalanine by the phenylalanine ammonia-lyase (PAL) $[9,10]$ that produces cinnamic acid. Cinnamic acid is then hydroxylated by the enzyme cinnamate-4-hydroxylase $(\mathrm{C} 4 \mathrm{H})$ producing $p$ coumaric acid [11], which is in turn activated by 4-coumarate:CoA ligase (4CL) to produce $p$-coumaroyl-CoA $[12,13]$. This product is processed by cinnamoyl-CoA reductase (CCR) to coniferaldehyde, which in turn is converted to coniferyl alcohol by the action of CAD. p-coumaroyl-CoA can also be transformed to $p$-coumaroyl-CoA shikimate by the action of hydroxycinamoyl transferase (HCT). p-coumaroyl-CoA shikimate proceeds through a series of transformations into caffeoyl shikimate, caffeoylCoA, feruloyl CoA, and coniferaldehyde by the action of the enzymes $p$-coumarate 3-hydrolase $(\mathrm{C} 3 \mathrm{H}), \mathrm{HCT}$, caffeoyl-CoA O-methyltransferase (CCOMT), and cinnamoyl CoA reductase (CCR), respectively. Coniferaldehyde can be transformed to coniferyl alcohol by the action of CAD or lead to 5-Hydroxy- coniferaldehyde and sinapyl aldehyde under the action of ferulate 5-hydrolase ( $55 \mathrm{H})$ and caffeic/5-hydroxyferulic acid O-methyltransferase
(COMT). The sinapyl alcohol is produced either from sinapyl aldehyde by CAD or from coniferyl alcohol by F5H and COMT. It has also been reported that the synthesis of sinapyl alcohol can be catalyzed by sinapyl alcohol dehydrogenase (SAD) [14]. However, recent studies $[15,16]$ did not find any detectable sinapyl alcohol dehydrogenase activity in Arabidopsis and Oryza indicating that the same CAD gene products can synthesize both coniferyl and sinapyl alcohols.

Because of its economic importance and biological role in various developmental and defense processes, the function of lignin biosynthesis related genes has been well studied in various plants $[17,18]$. Down-regulation of genes involved in the early steps of the monolignol synthesis pathway can lead to a reduction in lignin biosynthesis [17]. However, altered expression of CAD genes in various plants resulted in only slight variations in lignin content [19-23]. This is mainly due to the incorporation of other phenolic products that compensate for monolignols in lignin as well as the compensation by other members of the CAD gene family. A significant reduction of lignin was detected in natural CAD mutants in Pinus (5\%) and the $b m 2, b m 3$, and $b m 4$ mutants in maize (20\%) $[24,25]$. The gene underlying the $b m 1$ mutant in maize is not a CAD gene, however, and may encode a regulator of several CAD genes. Down-regulating the expression of CAD genes in Nicotiana tabacum, Populus, and Pinus showed no gross morphological variations but CAD deficient plants were enriched in coniferyl aldehyde and sinapyl aldehyde [24,26,27]. The accumulation of the aldehyde molecules is responsible for the red-brown color in the stems of natural and induced CAD mutants in Populus, Zea, Oryza, and Pinus $[15,16,24,25]$. A recent study in Arabidopsis showed that double mutants in the two major CAD genes associated with lignin biosynthesis (AtCAD_C and $A t C A D \_D$ named AtCAD4 and AtCAD5) present prostrate stems because of the weakness of the vasculature [15]. A reduction in the size and the diameter of the stems was also observed in the double mutant plants. Beside its role in plant development, CAD also seems to play a key role in plant defense against abiotic and biotic stresses $[1,28,29]$.

CAD proteins are encoded by a gene family in plants $[29,30]$. Complete sets of CAD genes and CAD-like genes 
have been previously identified in the genomes of model species (Arabidopsis, Oryza, and Populus) and partially from expressed sequences of non-model plants. In Arabidopsis, CAD exists as a multigene family consisting of nine genes (AtCAD1 to AtCAD9) [31,32]. Although all nine have been classified as CAD genes based on their predicted protein sequences, only CAD-C (AtCAD5) and CAD-D (AtCAD4) have been shown to have major roles in lignin synthesis in Arabidopsis [32,33]. AtCAD7 and AtCAD8 may also be involved to some extent in lignin biosynthesis [33]. AtCAD2, AtCAD3, AtCAD6, and AtCAD9 appear to encode mannitol dehydrogenases. A double mutation of AtCAD2 and AtCAD6 led to an overexpression of $A t C A D 1$ (AtCAD7) suggesting a compensation between some CAD genes [34]. In Oryza, 12 CAD genes have been reported [16].

Phylogenetic analysis [29,35] of the predicted amino acid sequences of CAD genes in Arabidopsis has shown that $\mathrm{CAD}$ is organized into three classes with gymnosperm sequences clustering in a separate group [29]. On the contrary, another study [30] showed that CAD genes were distributed in two classes both containing monocot and eudicot genes. The contradictory results obtained in these two studies were obtained using a limited set of genes and were not conclusive.

In this study we retrieved and compared CAD sequences from a wide variety of plants, making full use of the available plant genome sequences (Arabidopsis, Oryza, Populus, Medicago, and Vitis) as well as expressed sequence databases for species of basal angiosperms, gymnosperms, and mosses. This dataset was used to analyze the phylogeny of the CAD gene family. We also analyzed the organization, the structure, and the expression of CAD genes in Populus. This provided insight into the evolution of their structure and function as well as mechanisms that contributed to gene duplications.

\section{Results}

\section{CAD gene family organization}

In model species for which the genome is completely sequenced, $71 \mathrm{CAD}$ genes have been identified to date (see Additional file 1): 9 in Arabidopsis [36], 12 in Oryza [30], 15 in Populus (this study), 18 in Vitis (this study), and 17 in Medicago (this study). Furthermore, we identified 54 more CAD genes in 31 other species, which include a variety of eudicots, monocots, basal angiosperms, and gymnosperms. Additional file 1 includes the list of these CAD gene names based on the standard established by the International Populus Genome Consortium (IPGC)[35] with the names of species (Poptr for Populus trichocarpa for example), the protein name (CAD), and a designation of family and clade memberships derived from this study. Additional file 1 also pro- vides the accession number and database source for each gene.

Analysis of the physical gene distribution in the Arabidopsis and Populus genomes showed that most CAD genes were located on duplicated blocks. In Arabidopsis only one gene (AtCAD5) is not located on duplicated chromosomal blocks. Almost all of the genes are still in conserved positions within the duplicated blocks. In Populus, we found 14 of the 15 CAD genes distributed on duplicated regions. The Populus CAD genes were distributed on seven chromosomes with chromosomes I, IX, and XVI having three or more genes each (Fig. 1). PoptrCAD9 was located on a scaffold not yet assigned to a chromosome (see Additional file 1). Homologous pairs from the nine duplicated genes (PoptrCAD6, PoptrCAD11, PoptrCAD3, PoptrCAD4, PoptrCAD15, PoptrCAD16, PoptrCAD8, PoptrCAD2, and PoptrCAD5) remain in conserved positions on homeologous duplicated blocks. Duplicates of PoptrCAD1, PoptrCAD12, PoptrCAD7, and PoptrCAD14 appear to be lost from the Populus genome by an unknown gene death mechanism. PoptrCAD8, PoptrCAD16, and PoptrCAD15 seem to be generated via tandem duplications from one of the genes. Only PoptrCAD13 and PoptrCAD10 were not located on duplicated blocks.

In Oryza five CAD genes (OsCAD2, OsCAD9, OsCAD10, OsCAD11, and OsCAD8) were located on duplicated segments. Four CAD genes in rice (OsCAD8A, OsCAD8B, $O s C A D 8 C$, and OsCAD8D) were distributed one after the other at the same locus [30] indicating a possible tandem duplication origin.

\section{Intron-exon structure of CAD genes}

Gene structure analysis of Populus CAD genes (Fig. 2) revealed the existence of three patterns of intron-exon structures. Pattern 1 (PoptrCAD5, PoptrCAD10, PoptrCAD3, PoptrCAD9, PoptrCAD1， PoptrCAD13, PoptrCAD8, PoptrCAD6, PoptrCAD15, and PoptrCAD16), pattern 2 (PoptrCAD4), and pattern 3 (PoptrCAD2, PoptrCAD11, PoptrCAD12, PoptrCAD14, and PoptrCAD7) were composed by 5, 5, and 6 exons, respectively. Pattern 1 and pattern 2 present a difference in length of exon 3 and exon 4 . Genes within these patterns present a similar number and size of exons. All Populus duplicated genes show a similar structure. PoptrCAD16 and PoptrCAD8, which may have risen from PoptrCAD15 by tandem duplication, also showed the same structure. While the intron length is conserved between some homeologous introns, others exhibit a great deal of variation. The increase in length could be due to transposable element insertions. Homeologous duplicate pairs (PoptrCAD11 - PoptrCAD2, PoptrCAD5 - PoptrCAD3, and PoptrCAD6 - PoptrCAD8) genes also show similar structure between homologs (Fig. 2). 


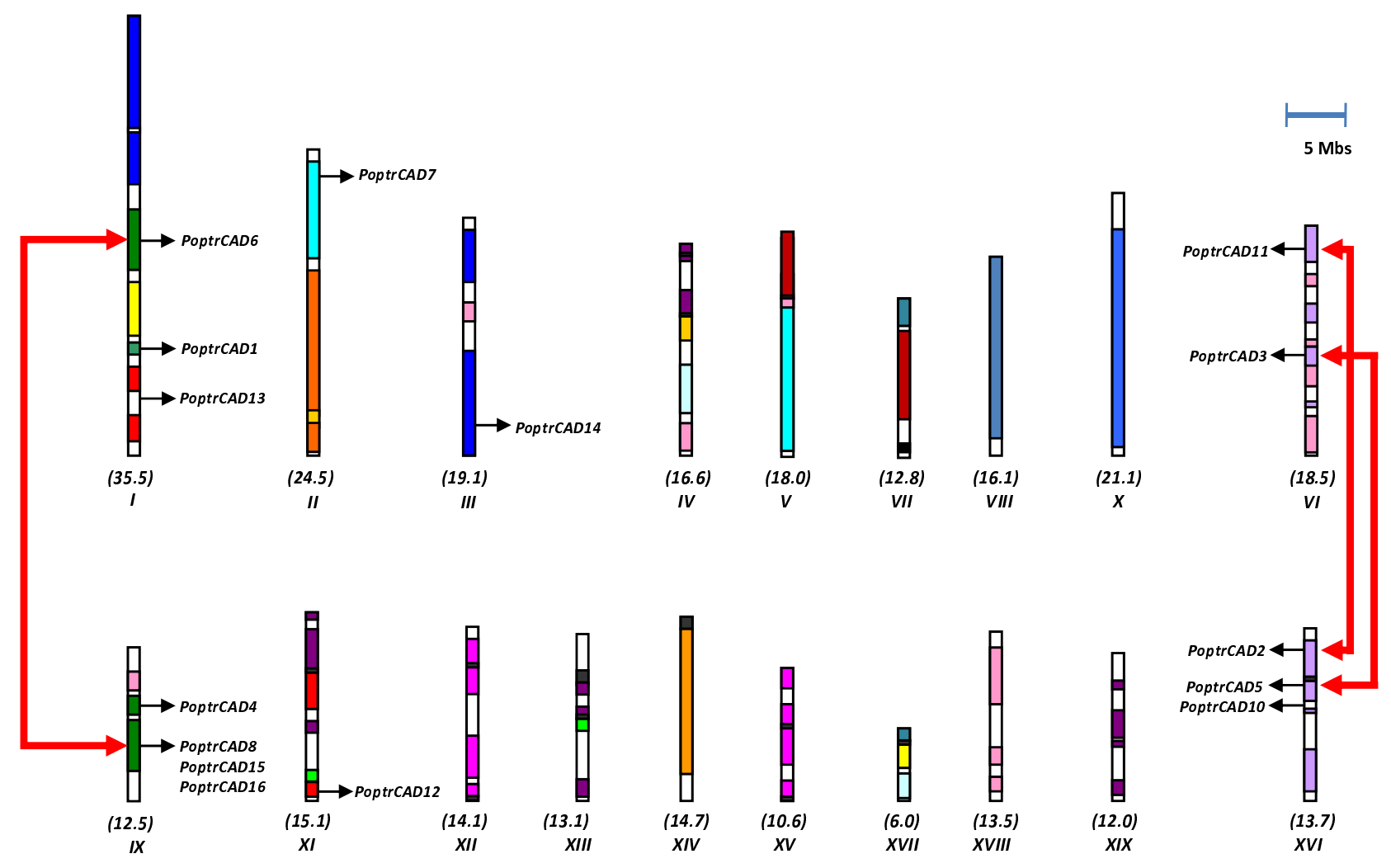

Figure I

Distribution of CAD genes on Populus chromosomes. The names of the chromosomes and their sizes (Mb) are indicated below each chromosome. Segmental duplicated homeologous blocks [39] are indicated with the same color. The position of genes is indicated with an arrowhead.

The number of different intron/exon patterns for Populus (this study), Oryza [30], and Arabidopsis [31] totaled three, four, and six, respectively. Pattern 1 and pattern 3 of intron-exon structure were common to eudicots and monocots, while pattern 2 was found only in eudicots. It is important to note that Oryza has the greatest number of intron-exon structure variants even though rice has fewer CAD genes than Populus and apparently less overall chromosomal duplications.

\section{Promoter sequence analysis}

Analysis of promoter sequences of the Populus CAD genes allowed us to identify several motifs that are known to be involved in the regulation of gene expression in various developmental and physiological processes (Table 1 and see Additional file 2). Some of those motifs interact with known regulators of genes involved in lignin biosynthesis such as $M y b$ and Zinc finger genes [37]. The other motifs are involved in the response to various hormones involved in responses to biotic and abiotic stresses such as auxin, ethylene, abscisic acid (ABA), salicylic acid, and Methyl Jasmonate (MeJA) (Brill et al., 1999; Mur et al.,
1996; Yasuda et al., 2008; Lawrence et al., 2006). PoptrCAD4 and PoptrCAD10, which are both preferentially expressed in xylem, possess transcription factor binding motifs involved in development and in response to various stresses, but showed some differences in their sets of motifs and in the distribution of the motifs in their promoter regions. For instance, PoptrCAD4 has motifs involved in response to ABA, stress, MeJA, wounding, and light. Unlike PoptrCAD4, PoptrCAD10 has motifs that bind to $\mathrm{Myb}$ and zinc finger proteins or are involved in response to auxin. Some CAD genes such as PoptrCAD1, PoptrCAD2, PoptrCAD10, and PoptrCAD11 possess promoter motifs involved in the response to fungal elicitors. Other genes (PoptrCAD2, PoptrCAD4, PoptrCAD5, PoptrCAD7, PoptrCAD9, PoptrCAD10, PoptrCAD16) possess motifs involved in response to wounding, herbivore stress, as well as other stresses.

\section{Evolution of CAD genes}

Maximum Likelihood (ML) bootstrap trees (based on nt and $\mathrm{AA}$ alignments) indicate that the CAD genes of land plants consist of three classes (Fig. 3). The distribution of 


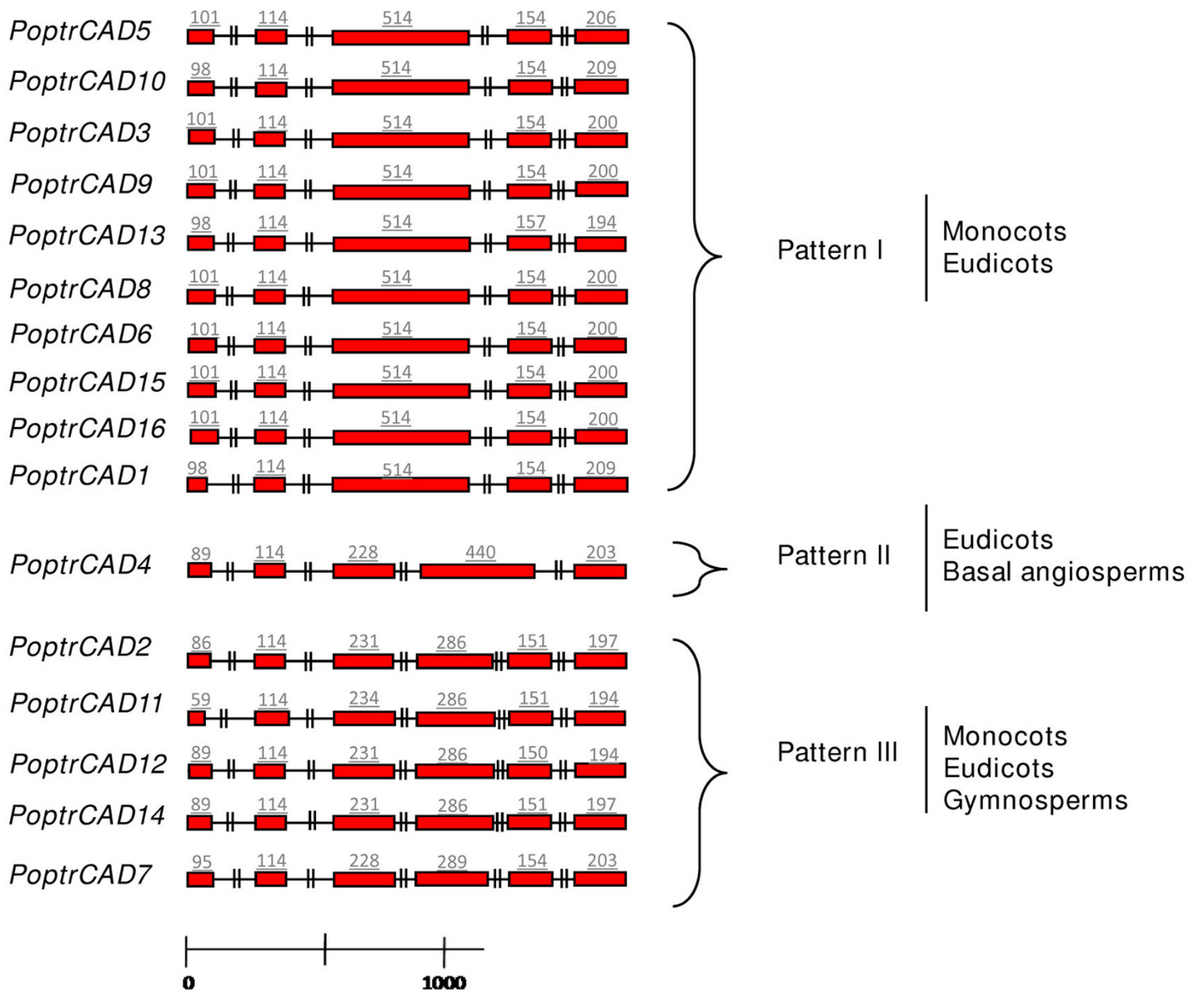

\section{Figure 2}

Intron-exon structures of CAD genes from Populus. Exons and introns are indicated by open boxes and lines respectively. Numbers above boxes indicate the exon sizes. The intron sizes are not to scale. The names of CAD genes and intronexon structure are indicated at the left and right sides respectively.

these three classes was supported by relatively high bootstrap values. Similar results were obtained using Neighbor joining (NJ) phylogenetic analyses (data not shown). Class I is represented by species from monocots, eudicots, and gymnosperms. Class II and Class III are represented by only sequences from angiosperms. The subdivision of Class I in two subclades is the result of a duplication event that happened in the ancestor of gymnosperms. The only known basal angiosperm (Saruma henryi) CAD (SheCAD_A) [38] is located in Class II. Class I contains the two Arabidopsis (AtCAD5 and AtCAD4) [32] CAD genes previously shown to be associated with lignin biosynthesis. It also includes PoptrCAD4 which we found to be pref- erentially expressed in xylem (this study). All the other genes from Populus trichocarpa and Arabidopsis were distributed in Class II and Class III. Clustering of several genes from monocots, eudicots, and gymnosperms suggest within-species duplications.

\section{Histochemistry of lignin deposition in P. trichocarpa tissues}

Before analyzing the expression of CAD genes using Real time RT-PCR, we analyzed lignin deposition patterns in the tissues of plants by staining with phloroglucinol and observation by light and fluorescent microscopy. The lignin distribution pattern under UV light was similar to 
Table I: List of motifs found in the promoter regions of Populus CAD genes.

\begin{tabular}{|c|c|c|c|c|c|c|c|c|c|c|c|c|c|}
\hline & $\begin{array}{l}\text { Salicylic } \\
\text { acid }\end{array}$ & Auxin & $\begin{array}{c}\text { Defense } \\
\text { /stress } \\
\text { responsi } \\
\text { veness }\end{array}$ & $\begin{array}{l}\text { Fungal } \\
\text { elicitor }\end{array}$ & $\begin{array}{c}\text { Methyl- } \\
\text { jasmonate }\end{array}$ & $\begin{array}{c}\text { Myb } \\
\text { binding }\end{array}$ & Wound & $\begin{array}{l}\text { Transcript } \\
\text { ion } \\
\text { Enhancer }\end{array}$ & $\begin{array}{c}\text { Zinc } \\
\text { finger } \\
\text { binding }\end{array}$ & Ethylene & $\begin{array}{c}\text { Herbivore } \\
\text { defense }\end{array}$ & $\begin{array}{c}\text { Abscisic } \\
\text { Acid }\end{array}$ & $\begin{array}{l}\text { Light } \\
\text { responsi } \\
\text { veness }\end{array}$ \\
\hline PoptrCADI & $\mathbf{x}$ & & & $\mathbf{x}$ & & & & & & $\mathbf{x}$ & & & $\mathbf{x}$ \\
\hline PoptrCAD2 & $\mathbf{x}$ & & & $\mathbf{x}$ & $\mathbf{x}$ & & $\mathbf{x}$ & & & & & & \\
\hline PoptrCAD3 & & $\mathbf{x}$ & $\mathbf{x}$ & & $\mathbf{x}$ & $\mathbf{x}$ & & $\mathbf{x}$ & $\mathbf{x}$ & & & & $\mathbf{x}$ \\
\hline PoptrCAD4 & $\mathbf{x}$ & & $\mathbf{x}$ & & $x$ & & $\mathbf{x}$ & & & & & $\mathbf{x}$ & $\mathbf{x}$ \\
\hline PoptrCAD5 & $x$ & & $\mathbf{x}$ & & $\mathbf{x}$ & & $x$ & & & & & & \\
\hline PoptrCAD6 & & $\mathbf{x}$ & & & & & & & $\mathbf{x}$ & $\mathbf{x}$ & & & $\mathbf{x}$ \\
\hline PoptrCAD7 & $\mathbf{x}$ & $x$ & & $\mathbf{x}$ & $\mathbf{x}$ & $\mathbf{x}$ & $\mathbf{x}$ & $\mathbf{x}$ & & & & & $\mathbf{x}$ \\
\hline PoptrCAD8 & $\mathbf{x}$ & $x$ & & & $x$ & $x$ & & & & & $\mathbf{x}$ & $\mathbf{x}$ & $\mathbf{x}$ \\
\hline PoptrCAD9 & $\mathbf{x}$ & $\mathbf{x}$ & & & & & $\mathbf{x}$ & $\mathbf{x}$ & $\mathbf{x}$ & & & & $\mathbf{x}$ \\
\hline PoptrCADIO & $\mathbf{x}$ & $\mathbf{x}$ & & & & & & & $\mathbf{x}$ & & & & \\
\hline PoptrCADII I & & & & $\mathbf{x}$ & $\mathbf{x}$ & & $\mathbf{x}$ & & & $\mathbf{x}$ & & $\mathbf{x}$ & \\
\hline PoptrCAD /2 & & & & & $\mathbf{x}$ & $\mathbf{x}$ & & & & & & $\mathbf{x}$ & \\
\hline PoptrCAD /3 & $\mathbf{x}$ & & & & & & & & & & $\mathbf{x}$ & & \\
\hline PoptrCAD /4 & & & & & $\mathbf{x}$ & & & $\mathbf{x}$ & & & & & $\mathbf{x}$ \\
\hline PoptrCAD I5 & & & $\mathbf{x}$ & & & & & & & & & & $\mathbf{x}$ \\
\hline PoptrCAD I6 & $\mathbf{x}$ & & & & & & $\mathbf{x}$ & & $\mathbf{x}$ & & & $\mathbf{x}$ & $\mathbf{x}$ \\
\hline
\end{tabular}

that of staining with acidified phloroglucinol, indicating that the same tissues were lignified. In leaf tissues lignin was detected mainly in the xylem of vascular bundles and in schlerenchyma fibers surrounding vascular tissues (Fig. $4 a, b)$. Petioles were lignified only in secondary cell walls of xylem and in the extensive hypodermal band of schlerenchyma (Fig. 4c, d). The most heavily lignified tissues were observed in stem segments. The bark of the stem, including phloem sieve tube cells, and parenchyma were not lignified (Fig. 4e). In the bark, lignin was detected only in schlerenchyma fibers at the outer part of phloem (Fig. 4e, f). Secondary xylem with thickened secondary cell walls showed the strongest reaction, demonstrating large amounts of lignin distributed in the tracheary vessels and fibers (Fig. 4g, h).

\section{Expression analysis of Populus CAD genes}

Of the 15 CAD genes found in Populus, we analyzed the expression of 13 (see Additional file 1) in several different tissues that were selected based on the previous histochemical studies (Fig. 4). Expression analysis using quantitative real-time RT-PCR (Fig. 5) showed that all CAD genes are expressed in leaves, petioles, bark and xylem, but at different levels among the tissues. PoptrCAD7, for example, is expressed in leaves and petioles, but presents a very low expression in the bark and xylem. The expression patterns vary widely between genes, which were sorted into four groups based on the expression profiles observed in different tissues (Fig. 3). Group 1 (PoptrCAD4; PoptrCAD10) is represented by genes strongly expressed in xylem (lignin associated) - 100 times more highly expressed in xylem than the other CAD genes. Statistical analysis using the Ward linkage method showed that group 1 is significantly different in expression from the other three groups. One-way ANOVA analysis showed that the expression of PoptrCAD4 and PoptrCAD10 (group 1 ) in the xylem was statistically different from each other ( $\mathrm{p}<0.005)$ with PoptrCAD10 more expressed. Group 2 (PoptrCAD13, PoptrCAD7, PoptrCAD12) genes are expressed in all tissues but are most highly expressed in leaves. The group 3 (PoptrCAD9) gene is preferentially expressed in leaves and xylem. Genes from group 4 (PoptrCAD2, PoptrCAD3, PoptrCAD5, PoptrCAD6, PoptrCAD11, PoptrCAD14, PoptrCAD15) did not show any significant expression differences between tissues. As indicated in Fig. 3, group 1 genes are distributed in Class I and Class II, group 2 and group 4 genes are distributed in Class II and Class III, while gene from group 3 belong to Class II.

Analysis of gene duplicates in Populus showed that PoptrCAD2 and PoptrCAD11 presented similar expression patterns in that they both did not show any significant expression differences between tissues. Similarly, PoptrCAD3 and PoptrCAD5 presented similar expression profiles in the tissues analyzed.

\section{Discussion}

\section{Organization of CAD genes in Populus}

Previous studies reported the identification of complete sets of CAD genes from the model plant species Arabidopsis and Oryza $[29,30]$, along with several sequences from non-model species [29,30,36]. Those studies [29,30,35] reported also preliminary phylogenetic trees for CAD genes based on a limited set of sequences mainly from Arabidopsis, Populus, and Oryza lineages. Moreover, no phylogenetic study including genome organization, gene structure, phylogeny, and expression profiling has been 


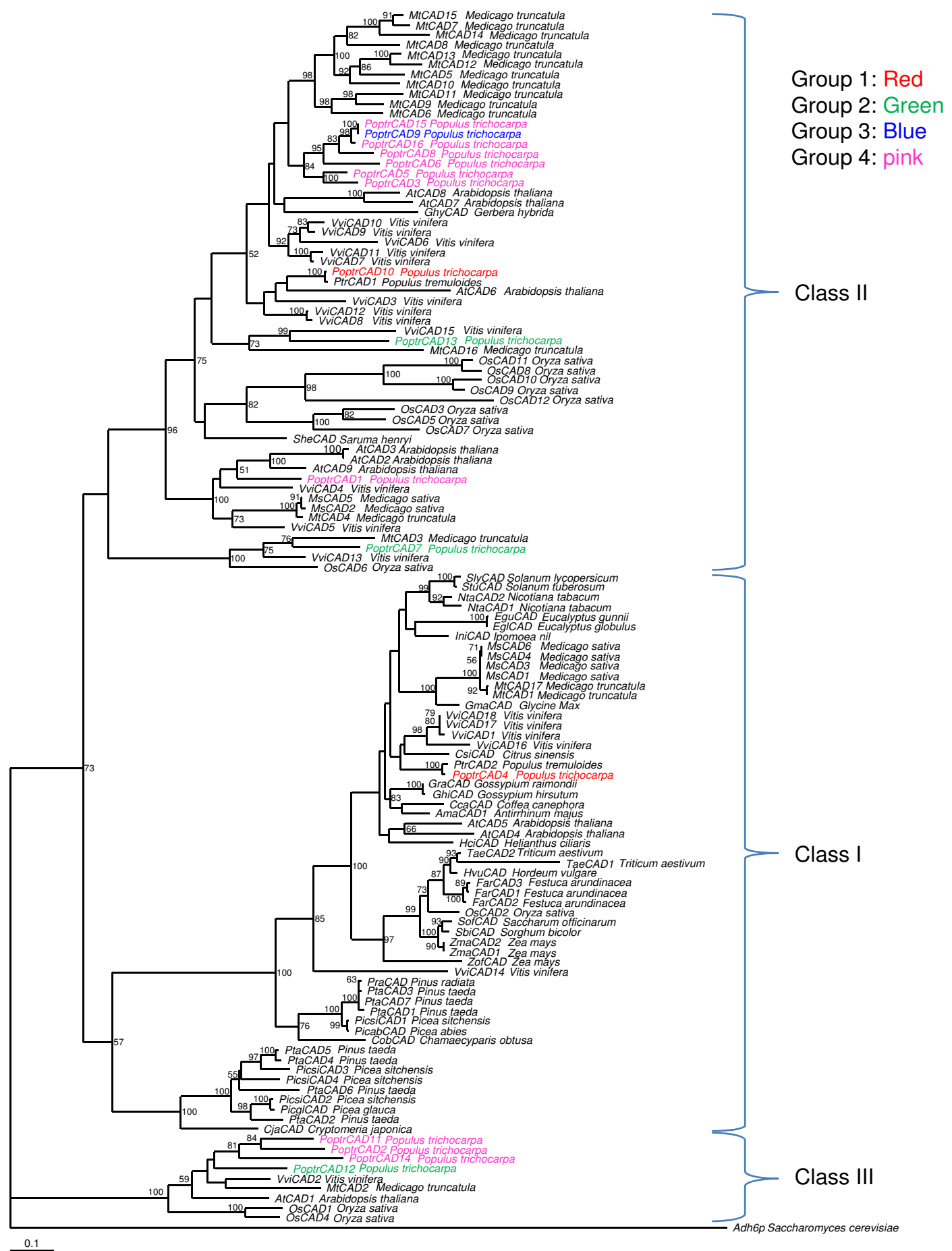

\section{Figure 3}

Maximum Likelihood bootstrap tree phylogeny based on amino acid sequences of CAD genes in various land plants. Numbers above branches refer to NJ bootstrap values. Brackets highlight the three classes of CAD genes. Colors indicate gene groups determined based on their expression in various Populus plant tissues. Red (group I), green (group 2), and blue (group 3) indicate genes preferentially expressed in xylem, leaves, as well as leaves and xylem respectively. Pink (group 4) represents genes that showed no preferential expression between Populus tissues. 

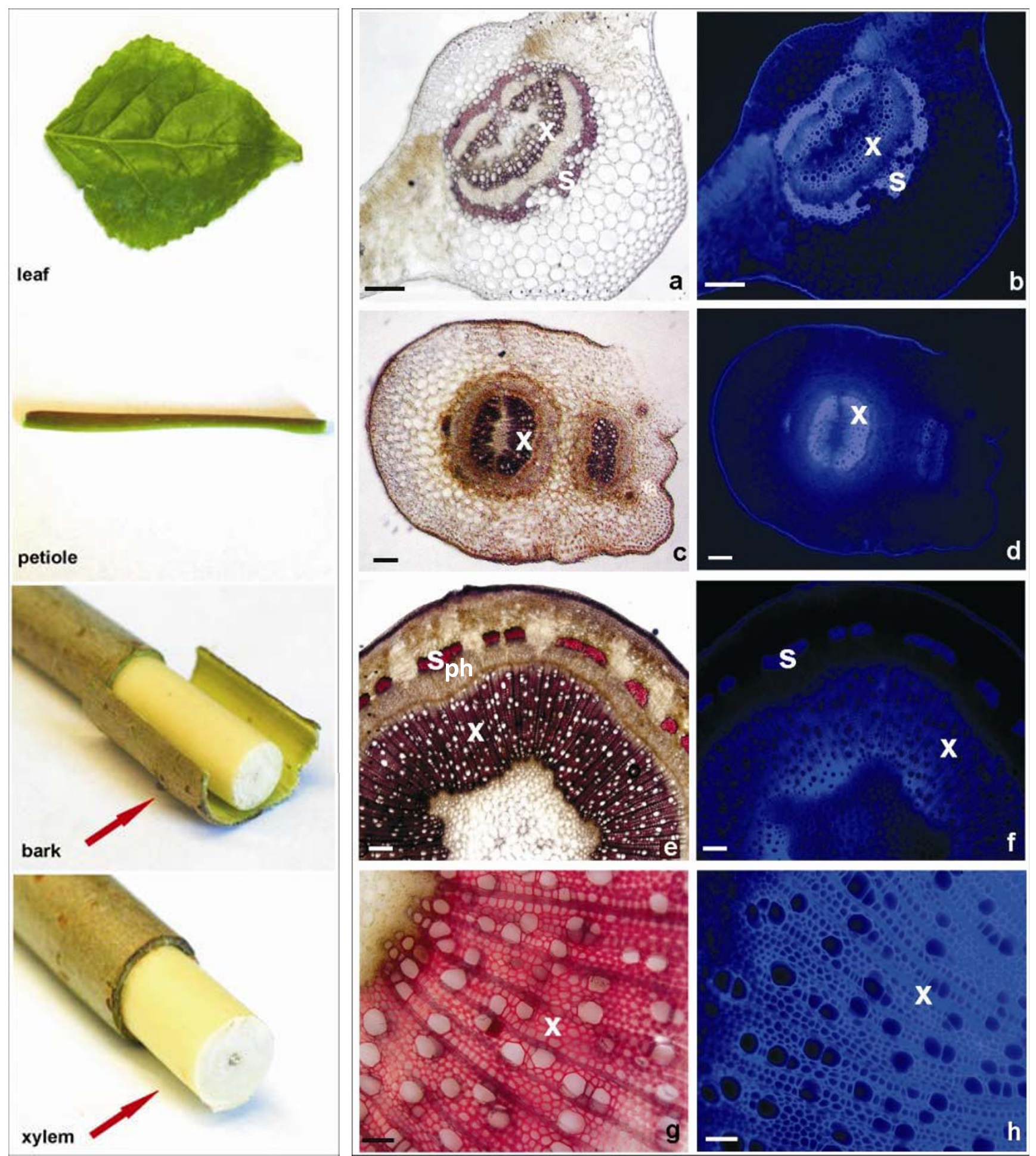

\section{Figure 4}

Lignification pattern in Populus tissues selected for qRT-PCR studies. Far left column displays organs and tissues used (Leaf blade, Petiole, bark, Xylem). Middle column shows lignin deposition, visualized under the light microscope after phloroglucine- $\mathrm{HCl}$ staining (red color). Right column shows lignin distribution by fluorescent microscopy (autofluorescence). a, b cross section of leaf vascular bundle, $c, d$ - petiole cross section, e, $f$ - transverse section of stem segment, $g, h-$ secondary xylem from stem. Abbreviations: $\mathrm{x}-\mathrm{xylem}, \mathrm{ph}-$ phloem, $\mathrm{s}-\mathrm{schlerenchyma}$. Bars $=100 \mu \mathrm{m}$. 


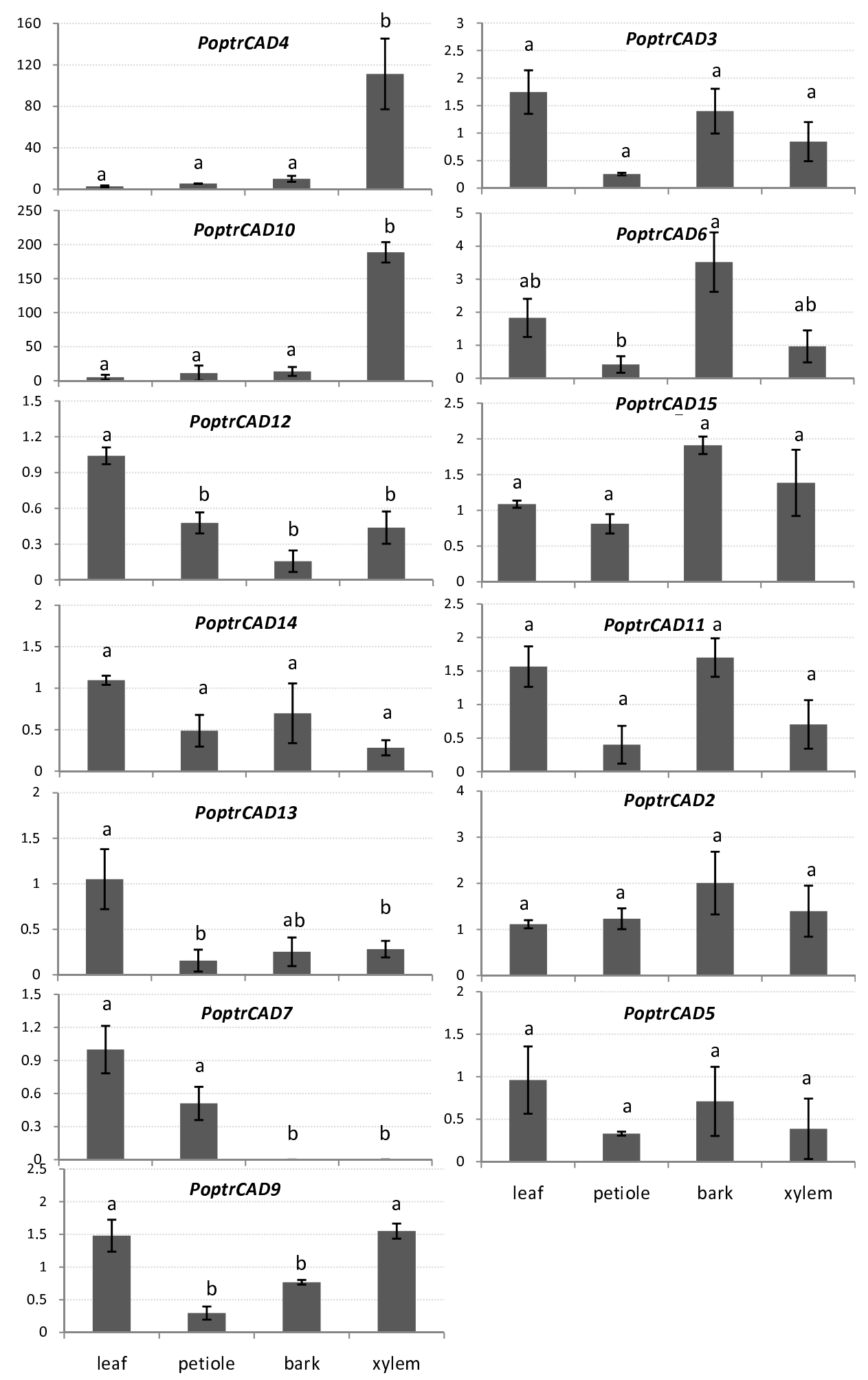

Figure 5

Quantitative expression of Populus CAD genes. The name of each gene is indicated at the top of each histogram. Tissues studied are shown at the bottom of the diagrams. Means designated by the same letter do not differ significantly according to Tukey's HSD test; $P<0.05)$. 
reported to date on the model tree species Populus. Here, we report the analysis of the phylogeny of CAD genes using five complete genome sequences and a set of genes from various land plant lineages. We also analyzed the structure of CAD genes and their promoters as well as their physical organization on Populus chromosomes and their expression patterns in various plant tissues.

Our study of the organization of CAD genes showed that chromosome duplications contributed significantly to the duplication of CAD genes in the Populus genome. Similar results were reported for Arabidopsis and Oryza [30,31]. Almost $80 \%$ of genes in Arabidopsis and Populus were distributed on duplicated regions. We cannot be sure if those duplications happened independently in both species or if some of them have occurred in the ancestor of those species. The distribution of several Populus duplicates on segmental duplications reported previously [35,39] associated with the Salicoid duplication event that occurred 65 million years (myrs) ago indicates that most CAD gene duplications happened in the ancestor of Populus. Dating duplications in Populus using a rate of $1.5 \times 10^{-8}$ synonymous substitutions per synonymous site per year as proposed by Koch et al., (2000) showed that most of them have occurred between 4 and 15 myrs ago. At least three other duplication events may have occurred prior to the large duplication event at $\sim 20, \sim 30$, and $~ 38$ myrs ago. This timing corresponds to the large duplication event reported previously ( 13 myrs) $[35,40]$ that occurred in the ancestor of Populus. However, based on the molecular clock timing, all duplication events seem to be postdating the earliest fossils of Populus, which are dated at 58-myr ago (Eckenwalder, 1996). The comparative timing of the duplication event reported in previous work [40] and in this study suggest that the timing of Populus duplications is not accurate as the Populus genome is evolving slowly compared to Arabidopsis. Nevertheless, the distribution of Populus CAD genes on segmental duplications associated with the Salicoid duplication, the agreement between our duplication timing result and those reported previously (Streck et al., 2005), and the distribution of CAD genes on the phylogenetic tree suggest that most of those duplications happened in the ancestor of Salicaceae. The retention of duplicate genes in the Populus genome is not surprising as the genome of this species has been suggested to evolve at a slow rate compared to Arabidopsis[35]. However, this retention seems to be common to several species such as Arabidopsis [36], Oryza [30,36], Populus (this tudy), Vitis (this study), and Medicago (this study). Whether the duplicated CAD genes correspond to genetic redundancy or have evolved divergent functions, they must be involved in important processes in the plant to be retained in these two very different eudicot species. In sharp contrast, only one rice CAD gene was found on a large duplicated block We are not sure if Oryza CAD genes did not experience large duplications or if most of the duplicates have already been lost. It is noteworthy that four Oryza CAD genes located at the same locus evidently evolved by inverted duplications. This may represent an alternative mechanism of CAD gene family evolution in rice versus Eurosids.

Three patterns of intron-exon structure were observed among CAD genes. Patterns 1 and 2 are characterized by 5 exons and 4 introns, while Pattern 3 CAD genes have 6 exons and 5 introns. Pattern 1 was detected in eudicots (Arabidopsis, Populus) and monocots (rice), while pattern 2 was found in eudicots (Arabidopsis and Populus) and a basal angiosperm, i.e Liriodendron tulipifera (Haiying Liang, personal communication). Pattern 3 was detected in eudicots and monocots (this study) as well as in gymnosperms [41]. Pattern 2 was found in several bona fide CAD genes (Class I) as well as some genes from Class II. Based on these results, at least pattern 2 and pattern 3 existed in the ancestor of angiosperms. This is confirmed by the dating of the duplication events of Populus genes, as the duplications that generated genes with pattern 1 were recent compared to the one that generated genes with pattern 2 and pattern 3. Furthermore, Oryza seems to have several other specific variant patterns of introns/exons that may have evolved in rice or the ancestor of the Poaceae, some lacking introns which were apparently generated by transposable elements. This diversification in rice could be linked to the high evolution rate of Poaceae genes compared to the two eudicot model species.

\section{CAD gene family is divided into three main classes}

Phylogenetic analyses showed that CAD genes are divided into three classes based on their $\mathrm{AA}$ and nt sequences. CAD class I included sequences from monocots, eudicots, and gymnosperms clades. Class II and Class III include sequences from monocots and eudicots. This indicates that the evolution of Class II and Class III happened in the ancestor of angiosperms, or at least prior to the split of monocots and dicots. This result is similar to the one published recently by Tuskan and collaborators [35] using mainly sequences from monocots and eudicots. The tree obtained in this study differs from previous analyses $[29,35]$ which grouped the CAD genes in Arabidopsis into three classes, with the gymnosperm sequences clustering in a separate class [29]. It is also different from the tree published previously [30] showing a distribution of CAD genes in two mains classes. The difference between our phylogeny and the ones published previously [29,30,35] could be due to the inclusion of a broader set of species in this study. Several sequences from various species cluster close to each other; suggesting that there are species- or lineage-specific CAD gene duplications. This is in accordance with the distribution of $\sim 80 \%$ of CAD genes from Arabidopsis and Populus on duplicated blocks, some of 
which may have been generated by lineage-specific duplications. It is noteworthy that except for the bona fide genes (AtCAD4 and AtCAD5) which belong to Class I, all the other Arabidopsis CAD genes (previously known as "CADlike genes") fell into Class II and Class III in our analysis. Other known bona fide CAD genes which were grouped into Class I in our study included Populus tremuloides PtrCAD_B (PtCAD) (Li et al., 2001), Oryza OsCAD2 (OsCAD2) (Tobias et al., 2005), and Eucalyptus Egu_A (EuCAD2 or EgCAD) (Grima-Pettennati et al., 1993). Populus tremuloides SAD gene (Li et al., 2001) and Arabidopsis genes (AtCAD4 and AtCAD5) [33], which were reported as being involved in lignin biosynthesis were located in class II in our study. PoptrCAD4 and PoptrCAD10, which were highly preferentially expressed in xylem, were found in Class I and Class II respectively. Based on the close distribution of PoptrCAD10 to Populus tremuloides SAD on the phylogenetic tree; it seems that PoptrCAD10 is the ortholog of Populus tremuloides SAD gene. This result confirms previous results (Li et al., 2001) showing that there are two genes (CAD and SAD) involved in lignin biosynthesis in xylem from Populus trichocarpa and Populus tremuloides. Class III is represented by ATCAD1 which was reported presenting similar expression profile as bona fide genes (AtCAD4 and AtCAD5) in Arabidopsis plant tissues [33] even though their CAD catalytic activity could not be proven.

Previous studies reported the distribution of CAD genes in several classes and suggest that with the exception of bona fide lignin biosynthesis genes, all others are involved in plant defense (Tuskan et al., 2006). The distribution of most bona fide CAD genes from various species in Class I in this study favors such a functional distinction between Class I and II genes. However, the exceptions of PoptrCAD10 (SAD) from Populus trichocarpa, PtrCAD1 (SAD) from Populus tremuloides, and AtCAD8 and AtCAD7 [33], which were reported as being lignin associated and are distributed in class II, rule against this hypothesis. The most probable hypothesis is that some genes from class II evolved a modified expression profile or function such as plant defense against pathogens. The gain of function hypothesis for the genes from Class II is supported by the fact that some genes from this class are still associated with lignin biosynthesis in xylem. Two alternate hypotheses could explain the evolution of defense function of CAD genes. The first hypothesis is that CAD genes evolved defense function after the split of Class II and Class III from Class I genes. The second hypothesis is that the functional divergence of CAD genes occurred before the split of Class II and Class III from Class I. Further functional analysis of genes from Class I and Class II will be needed to answer this question.

\section{CAD genes show different expression profiles in various Populus tissues and possibly divergent functions}

The high rate of duplication of CAD genes and the retention of most duplicates raises the question of their functional redundancy. Quantitative expression analysis showed that among the CAD genes studied, four expression patterns were presented in the tissues studied. PoptrCAD4 and PoptrCAD10 from expression-group 1 were differentially expressed in xylem tissues and are associated with lignin biosynthesis. This conclusion is supported by the distribution of PoptrCAD4 into Class I with several previously reported bona fide CAD genes [33]. PoptrCAD10 clusters in Class II closely with the Populus tremuloides SAD gene and Arabidopsis AtCAD8 and AtCAD7, which has been reported as being involved in lignin biosynthesis [14,33]. Promoter analysis (Table 1) showed that PoptrCAD4 possess several motifs involved in stress response such as defense/stress responsiveness, MeJA, ABA, and light responsiveness. In contrast, PoptrCAD10 possess motifs involved in the interaction with zinc finger binding transcription factor and in the response to auxin. This result suggests that while both genes are involved in lignin biosynthesis, PoptrCAD4 expression may be modulated under biotic stress conditions. Genes from expression-groups 2 and 3, which are preferentially expressed in leaves could correspond to a defense-related lignin- biosynthesis pathway or other defense pathway as suggested previously $[42,43]$. They possess motifs involved in response to herbivory, wound, and MeJA. MeJA plays a key role in plant defense against various biotic and abiotic stresses [44,45]. Preliminary expression profiling of these genes in Populus under stress conditions confirmed this hypothesis as some of those genes increase their expression under herbivore (Gypsy moth) stress (data not shown). This result is not surprising as most pathogen invasions occur in the leaves. It is also in accordance with previous studies showing that CAD-like genes are involved in plant defense [43]. CAD genes from expression-group 4, which did not present any expression difference between various plant tissues, possess several motifs that are involved in response to MeJA, wound, fungal elicitor, stress and defense responsiveness, and ethylene. Those genes may function in lignin biosynthesis under other stress conditions. Comparison of gain/ loss of motifs in the promoter region did not allow the identification of probable motifs underlying the difference in expression profiles between bona fide CAD genes and the CAD-like genes.

From a functional perspective, the lingering question is why diverse copies of CAD genes from Class II and Class III have been maintained within plant genomes. One can ask if CAD genes from Class II and Class III, except PoptrCAD10, are involved only in plant defense or some of them can still compensate the function of bona fide 
CAD genes (PoptrCAD4 and PoptrCAD10) in lignin biosynthesis in xylem. The expression profile differences between CAD-like genes from Class II and Class III in the various tissues analyzed, added to the differential distribution of several motifs involved in various developmental and physiological processes in their promoter regions suggests that there is a functional specialization of CAD genes in various tissues and under various development and stress conditions. Expression analysis of two pairs of paralogs (PoptrCAD3 and PoptrCAD5, PoptrCAD2 and PoptrCAD11) showed that they have similar expression profiles. This suggests that the duplication of those genes did not result in divergence of their expression profile and function. However, we cannot rule out this hypothesis as these duplicate genes present different motifs for responses to various stresses in their promoter regions. Moreover, the expression of those duplicate genes could be regulated at the protein level. Therefore, the quantification of protein corresponding to those genes is needed to confirm this hypothesis.

\section{Conclusion}

In conclusion, we identified 15 CAD genes in Populus and found that most of them were located in the genome on duplicated blocks. We demonstrated that CAD genes in land plants were distributed in three phylogenetic classes of which two may have originated from duplications in the ancestry of all angiosperms. Class I genes function in lignin biosynthesis in xylem while genes from Classes II and III may function under stresses conditions. Promoter sequence analysis and preliminary results on expression profiling of CAD genes in tissues suggest CAD genes have evolved divergent expression profiles or functions.

\section{Methods \\ CAD sequences used in phylogenetic analysis}

CAD sequences used in phylogenetic analyses (see Additional file 1) include sequences generated in this study as well as sequences retrieved from different databases. We used sequences from plants with fully sequenced genomes as well as other taxons representing key positions on the angiosperm phylogenetic tree. CAD sequences from Arabidopsis, Oryza, and Populus were retrieved from TAIR http://www.Arabidopsis.org/, TIGR http://www.tigr.org, and the Joint Genome Institute http://www.jgi.doe.gov. CAD sequences from the newly sequenced genomes of Carica papaya, Vitis vinifera, and Medicago truncatula were retrieved from The Hawaii Papaya Genome Project [46], the Vitis genome [47], and the Medicago Sequencing Resources http://www.medicago.org/genome/, respectively. CAD sequences from various non model species were retrieved from TIGR Plant Genomics databases http:/ /www.tigr.org, GeneBank http://www.ncbi.nlm.nih.gov TIGR http://www.tigr.org, and the floral genome project database [38] databases. Sequences were carefully inspected and corrected for annotation errors before use.

Intron-exon structure and promoter analysis of CAD genes The exon/inron structure of CAD genes was retrieved from the Joint Genome Institute http://www.jgi.doe.gov web site. For genes for which complementary DNA (cDNA) sequences were available, the structure is checked by aligning genomic and cDNA sequences. Promoter analysis was done by querying all CAD genes against TRANSFAC [48] and PlantCARE [49].

\section{CAD sequences alignment and phylogenetic analyses}

CAD nucleotide cDNA sequences were translated into protein sequences. The inferred protein sequences were then aligned using Muscle with default parameters [50], and manually adjusted. Phylogenetic analyses were performed on the aligned amino acid (AA) sequences, as well as on the nucleotide sequences that were aligned to match the AAa. The GTR model [51] was found to be optimal for nt datasets, assuming among site rate heterogeneity and a proportion of invariable sites $(\mathrm{GTR}+\mathrm{G}+\mathrm{I})$, while the WAG model [52], assuming among site rate heterogeneity $(\mathrm{WAG}+\mathrm{G})$, was found to be the best fit for the aa sequences. These models were used for Maximum Likelihood (ML) analyses implemented in PHYML v. 2.4.4 [53]. 250 bootstrap replicates were run to estimate branch support. Neighbor-joining (NJ) analyses were performed in MEGA4. Since the models of best fit were not available here, we chose the JTT and Tajima-Nei models, using pairwise deletion and assuming gamma distributed site rates. 500 bootstrap replicates were run to estimate branch support.

\section{Histochemistry of lignin deposition analyses}

For visualization of lignin distribution, plant material (leaf blades, petioles, and stem) was free-hand sectioned with a razor blade. Sections were stained with phloroglucinol $(2 \% \mathrm{w} / \mathrm{v}$ phloroglucinol acidified in $6 \mathrm{M} \mathrm{HCl})$, mounted in glycerol and observed under an Olympus BX51 light and fluorescent microscope, equiped with a SPOT II RT digital camera.

\section{RNA isolation and cDNA synthesis}

Leaves, petioles, stem secondary cortex and stem xylem were collected from young hybrid Populus OGY (P. deltoides $\times P$. nigra) young trees grown in a culture chamber at $25^{\circ} \mathrm{C}$ and $18^{\circ} \mathrm{C}$ in the day and night, respectively. The plants were grown at $16 \mathrm{~h} / 8 \mathrm{~h}$ day/night regime and at $60 \%$ humidity. Tissues were harvested and immediately frozen in liquid nitrogen and stored at $-80^{\circ} \mathrm{C}$ until used for RNA isolation. Total RNA was isolated using CTAB method [54] with minor modifications. The RNA quality and concentration was assessed using an Agilent 2100 Bioanalyzer (Agilent Technologies). To remove any con- 
taminating genomic DNA, RNA samples were treated with RNAse free DNAse (Applied Biosystems) before real time RT-PCR experiments. RNA was reverse transcribed using random primers from the High Capacity cDNA Reverse Transcription kit (Applied Biosystems) and random primers following the manufacturer's recommendations. One microgram of total RNA from each sample was reversetranscribed to generate cDNA.

\section{CAD expression analysis using quantitative real time $R T$ - PCR}

Quantitative real time PCR reactions were prepared using the SYBR Green Master Mix kit (Applied Biosystems) and performed in an Applied Biosystems 7500 Fast Real-Time PCR system (Applied Biosystems) with default parameters. Primers used in this study (see Additional file 3) were designed using Primer Express ${ }^{\circledR}$ software (Applied Biosystems) or primer 3 software (The Whitehead Institute for Biomedical Research, Cambridge, MD, USA). We used the gene encoding the $18 \mathrm{~S}$ rRNA as an endogenous control to normalize for template quantity. The real-time PCR protocol was performed as following: denaturation by a hot start at $95^{\circ} \mathrm{C}$ for $10 \mathrm{~min}$, followed by 40 cycles of a twostep program (denaturation at $95^{\circ} \mathrm{C}$ for $15 \mathrm{sec}$ and annealing/extension at $60^{\circ} \mathrm{C}$ for $1 \mathrm{~min}$ ). Dissociation curves were used to verify the specificity of PCR amplification. For each tissue, samples from three different trees were used. Triplicate experiments were analyzed for each tissue and each tree. Data was evaluated using the 7500 Fast System SDS software procedures (Applied Biosystems). Statistical analyses were performed using Statistica 6.0 software (StatSoft Poland Inc., Tulsa, OH, USA).

\section{Abbreviations}

CAD: Cinnamyl alcohol dehydrogenase; nt: nucleotide; AA: amino acids; cDNA: complementary DNA; RT-PCR: Reverse transcriptase polymerase chain reaction; PAL: phenylalanine ammonia-lyase; HCT: hydroxycinnamoyl:CoA shikimate/quinate hydroxycinnamoyl transferase; 4CL: 4-coumarate:CoA ligase; CCR : cinnamoylCoA reductase; $\mathrm{C} 3 \mathrm{H}$ : p-coumarate 3-hydrolase.

\section{Authors' contributions}

$\mathrm{AB}$ retrieved, curated, annotated, and aligned the CAD nucleotide and protein sequences. He analyzed the gene structure, ran the phylogenetic analyses, supervised ABZ, AC, UP, SD, and PY, and wrote the manuscript. ABZ contributed to the RNA preparation and the expression analyses. UP and AC contributed to curating and aligning the CAD sequences. SD collected Populus tissues and participated in RNA preparation. PY contributed to promoter sequence analysis. This project was initiated by $\mathrm{AB}$ and JC. JC directs The Schatz Center for Tree Molecular Genetics at Penn State which funded the project, and he contributed to the evaluation and discussion of the results, and assisted in the preparation of the manuscript. All authors read and approved the final manuscript.

\section{Additional material}

\section{Additional file 1}

List of plant genes used in CAD gene phylogenetic analyses. The gene names used in this study, the accession number, species, the database source, and names of previously published genes are indicated. Click here for file

[http://www.biomedcentral.com/content/supplementary/14712229-9-26-S1.xls]

\section{Additional file 2}

List and description of nucleotide motifs discovered in the promoter regions of Populus CAD genes.

Click here for file

[http://www.biomedcentral.com/content/supplementary/14712229-9-26-S2.xls]

\section{Additional file 3}

List of Populus trichocarpa primers used for expression profiling of CAD genes in hybrid Populus (P. deltoides $\times$ P. nigra).

Click here for file

[http://www.biomedcentral.com/content/supplementary/14712229-9-26-S3.xls]

\section{Acknowledgements}

The authors thank The Joint Genome Institute for providing access to Populus trichocarpa genome sequences and the Electron Microscopy Facility at Pennsylvania State University for providing access to the microscope. We thank Dr. Claude dePamphilis for advice about phylogenetic analyses. We also thank Dr. Dawn Luthe for access to the real time RT-PCR machine and Yang Han for her help in analyzing the RT-PCR results. Many thanks to our colleagues Chris Frost for providing us with Populus plants and Teodora Best with advice on statistical analysis. This work was supported by The Schatz Center for Tree Molecular Genetics at Penn State.

\section{References}

I. Kiedrowski S, Kawalleck P, Hahlbrock K, Somssich IE, Dangl JL: Rapid activation of a novel plant defense gene is strictly dependent on the Arabidopsis RPMI disease resistance locus. The EMBO Journal 1992, I I ( I3):4677-4684.

2. Lao M, Arencibia AD, Carmona ER, Acevedo R, Rodriguez E, Leon $O$, Santana I: Differential expression analysis by cDNA-AFLP of Saccharum spp. after inoculation with the host pathogen Sporisorium scitamineum. Plant Cell Rep 2008, 27(6): I I03-I I I I.

3. Ithal N, Recknor J, Nettleton D, Maier T, Baum TJ, Mitchum MG: Developmental transcript profiling of cyst nematode feeding cells in soybean roots. Mol Plant Microbe Interact 2007, 20(5):510-525.

4. Zabala G, Zou J, Tuteja J, Gonzalez DO, Clough SJ, Vodkin LO: Transcriptome changes in the phenylpropanoid pathway of Glycine max in response to Pseudomonas syringae infection. BMC Plant Biol 2006, 6:26.

5. Donaldson L, Hague J, Snell R: Lignin Distribution in Coppice Poplar, Linseed and Wheat Straw. Holzforschung 200I, 55(4):379-385.

6. Higuchi T: Lignin Biochemistry: Biosynthesis and Biodegradation. Wood Sci Technol 1990, 24:23-63.

7. Whetten RW, MacKay JJ, Sederoff RR: Recent advances in understanding lignin biosynthesis. Annu Rev Plant Physiol Plant Mol Biol 1998, 49:585-609. 
8. Hoffmann L, Besseau S, Geoffroy P, Ritzenthaler C, Meyer D, Lapierre $C$, Pollet $B$, Legrand $M$ : Silencing of hydroxycinnamoyl-coenzyme A shikimate/quinate hydroxycinnamoyltransferase affects phenylpropanoid biosynthesis. Plant Cell 2004, 16(6): | 1446-1465.

9. Elkind Y, Edwards R, Mavandad M, Hedrick SA, Ribak O, Dixon RA Lamb C]: Abnormal plant development and down-regulation of phenylpropanoid biosynthesis in transgenic tobacco containing a heterologous phenylalanine ammonia-lyase gene. Proc Natl Acad Sci USA 1990, 87(22):9057-9061.

10. Bate NJ, Orr J, Ni W, Meromi A, Nadler-Hassar T, Doerner PW, Dixon RA, Lamb C], Elkind Y: Quantitative relationship between phenylalanine ammonia-lyase levels and phenylpropanoid accumulation in transgenic tobacco identifies a rate-determining step in natural product synthesis. Proc Natl Acad Sci USA 1994, 9 I(16):7608-76I2.

II. Sewalt V, Ni W, Blount JW, Jung HG, Masoud SA, Howles PA, Lamb C, Dixon RA: Reduced Lignin Content and Altered Lignin Composition in Transgenic Tobacco Down-Regulated in Expression of L-Phenylalanine Ammonia-Lyase or Cinnamate 4-Hydroxylase. Plant Physiol I997, I I 5(I):4I-50.

12. Kajita S, Katayama $Y$, Omori S: Alterations in the biosynthesis of lignin in transgenic plants with chimeric genes for 4-coumarate: coenzyme A ligase. Plant Cell Physiol 1996, 37(7):957-965.

13. Lee D, Meyer K, Chapple C, Douglas CJ: Antisense suppression of 4-coumarate:coenzyme $A$ ligase activity in Arabidopsis leads to altered lignin subunit composition. Plant Cell 1997, 9(I I): 1985-1998.

14. Li L, Cheng XF, Leshkevich J, Umezawa T, Harding SA, Chiang VL: The last step of syringyl monolignol biosynthesis in angiosperms is regulated by a novel gene encoding sinapy alcohol dehydrogenase. Plant Cell 200 I, I3(7): I567-I586.

15. Jourdes M, Cardenas CL, Laskar DD, Moinuddin SG, Davin LB, Lewis NG: Plant cell walls are enfeebled when attempting to preserve native lignin configuration with poly-p-hydroxycinnamaldehydes: evolutionary implications. Phytochemistry 2007, 68(14): 1932-1956.

16. Zhang K, Qian Q, Huang Z, Wang Y, Li M, Hong L, Zeng D, Gu M, Chu C, Cheng Z: GOLD HULL AND INTERNODE2 encodes a primarily multifunctional cinnamyl-alcohol dehydrogenase in rice. Plant Physiol 2006, I40(3):972-983.

17. Boerjan W, Ralph J, Baucher M: Lignin biosynthesis. Annu Rev Plant Biol 2003, 54:5 19-546.

18. Dixon RA, Chen F, Guo D, Parvathi K: The biosynthesis of monolignols: a "metabolic grid", or independent pathways to guaiacyl and syringyl units? Phytochemistry 200I, 57(7): 1069-1084.

19. Baucher M, Bernard-Vailhe MA, Chabbert B, Besle JM, Opsomer C Van Montagu M, Botterman J: Down-regulation of cinnamyl alcohol dehydrogenase in transgenic alfalfa (Medicago sativa $L$.) and the effect on lignin composition and digestibility. Plant Mol Biol I 999, 39(3):437-447.

20. Baucher M, Chabbert B, Pilate G, Van Doorsselaere J, Tollier MT, Petit-Conil M, Cornu D, Monties B, Van Montagu M, Inze D, Jouanin L, Boerjan W: Red Xylem and Higher Lignin Extractability by Down- Regulating a Cinnamyl Alcohol Dehydrogenase in Poplar. Plant Physiology 1996, I I 2: I 479-1490.

21. Lapierre C, Pollet B, Petit-Conil M, Toval G, Romero J, Pilate G, Leple JC, Boerjan W, Ferret V, De Nadai V, Jouanin L: Structural alterations of lignins in transgenic poplars with depressed cinnamyl alcohol dehydrogenase or caffeic acid O-methyltransferase activity have an opposite impact on the efficiency of industrial kraft pulping. Plant Physiol 1999, I I 9(I): I53-164.

22. Pilate G, Guiney E, Holt K, Petit-Conil M, Lapierre C, Leple JC, Pollet B, Mila I, Webster EA, Marstorp HG, Hopkins DW, Jouanin L, Boerjan W, Schuch W, Cornu D, Halpin C: Field and pulping performances of transgenic trees with altered lignification. Nat Biotechnol 2002, 20(6):607-6I2.

23. Sederoff RR, MacKay J], Ralph J, Hatfield RD: Unexpected variation in lignin. Curr Opin Plant Biol I999, 2(2): I 45-152.

24. MacKay JJ, O'Malley DM, Presnell T, Booker FL, Campbell MM, Whetten RW, Sederoff RR: Inheritance, gene expression, and lignin characterization in a mutant pine deficient in cinnamyl alcohol dehydrogenase. Proc Natl Acad Sci USA 1997, 94(I5):8255-8260.
25. Guillaumie S, Pichon M, Martinant JP, Bosio M, Goffner D, Barriere Y: Differential expression of phenylpropanoid and related genes in brown-midrib bm I, bm2, bm3, and bm4 young near-isogenic maize plants. Planta 2007, 226(I):235-250.

26. Kim H, Ralph J, Lu F, Pilate G, Leple JC, Pollet B, Lapierre C: Identification of the structure and origin of thioacidolysis marker compounds for cinnamyl alcohol dehydrogenase deficiency in angiosperms. J Biol Chem 2002, 277(49):474I2-474I9.

27. Ralph J, Lapierre C, Marita JM, Kim H, Lu F, Hatfield RD, Ralph S, Chapple C, Franke R, Hemm MR, Van Doorsselaere J, Sederoff RR, O'Malley DM, Scott JT, MacKay JJ, Yahiaoui N, Boudet A, Pean M, Pilate G, Jouanin L, Boerjan W: Elucidation of new structures in lignins of CAD- and COMT-deficient plants by NMR. Phytochemistry 2001, 57(6):993-1003.

28. Mitchell HJ, Hall JL, Barber MS: Elicitor-Induced Cinnamyl Alcohol Dehydrogenase Activity in Lignifying Wheat (Triticum aestivum L.) Leaves. Plant Physiol 1994, I04(2):55 I-556.

29. Raes J, Rohde A, Christensen JH, Peer $Y$ Van de, Boerjan $W$ : Genome-wide characterization of the lignification toolbox in Arabidopsis. Plant Physiol 2003, 133(3): 105 I-107I.

30. Tobias CM, Chow EK: Structure of the cinnamyl-alcohol dehydrogenase gene family in rice and promoter activity of a member associated with lignification. Planta 2005, 220(5):678-688.

3I. Tavares R, Aubourg S, Lecharny A, Kreis M: Organization and structural evolution of four multigene families in Arabidopsis thaliana: AtLCAD, AtLGT, AtMYST and AtHD-GL2. Plant Mol Biol 2000, 42(5):703-7I7.

32. Sibout R, Eudes A, Pollet B, Goujon T, Mila I, Granier F, Seguin A, Lapierre $C$, Jouanin L: Expression pattern of two paralogs encoding cinnamyl alcohol dehydrogenases in Arabidopsis. Isolation and characterization of the corresponding mutants. Plant Physiol 2003, I32(2):848-860.

33. Kim SJ, Kim KW, Cho MH, Franceschi VR, Davin LB, Lewis NG: Expression of cinnamyl alcohol dehydrogenases and their putative homologues during Arabidopsis thaliana growth and development: lessons for database annotations? Phytochemistry 2007, 68(14): 1957-1974.

34. Sibout R, Eudes A, Mouille G, Pollet B, Lapierre C, Jouanin L, Seguin $A$ : Cinnamyl alcohol dehydrogenase-C and $-D$ are the primary genes involved in lignin biosynthesis in the floral stem of Arabidopsis. Plant Cell 2005, I 7(7):2059-2076.

35. Tuskan GA, Difazio S, Jansson S, Bohlmann J, Grigoriev I, Hellsten U, Putnam N, Ralph S, Rombauts S, Salamov A, et al.: The genome of black cottonwood, Populus trichocarpa (Torr. \& Gray). Science 2006, 3 I3(5793): 1596-1604.

36. Kim SJ, Kim MR, Bedgar DL, Moinuddin SG, Cardenas CL, Davin LB, Kang C, Lewis NG: Functional reclassification of the putative cinnamyl alcohol dehydrogenase multigene family in Arabidopsis. Proc Natl Acad Sci USA 2004, I 0 I (6): I 455- I 460.

37. Goicoechea M, Lacombe E, Legay S, Mihaljevic S, Rech P, Jauneau A, Lapierre C, Pollet B, Verhaegen D, Chaubet-Gigot N, Grima-Pettenati J: EgMYB2, a new transcriptional activator from Eucalyptus xylem, regulates secondary cell wall formation and lignin biosynthesis. Plant / 2005, 43(4):553-567.

38. Albert VA, Soltis DE, Carlson JE, Farmerie WG, Wall PK, llut DC Solow TM, Mueller LA, Landherr LL, Hu Y, Buzgo M, Kim S, Yoo MJ, Frohlich MW, Perl-Treves R, Schlarbaum SE, Bliss B], Zhang X, Tanksley SD, Oppenheimer DG, Soltis PS, Ma H, DePamphilis CW, Leebens-Mack JH: Floral gene resources from basal angiosperms for comparative genomics research. BMC Plant Biol 2005, 5:5.

39. Kalluri UC, Difazio SP, Brunner AM, Tuskan GA: Genome-wide analysis of Aux/IAA and ARF gene families in Populus trichocarpa. BMC Plant Biol 2007, 7:59.

40. Sterck L, Rombauts S, Jansson S, Sterky F, Rouze P, Peer Y Van de: EST data suggest that poplar is an ancient polyploid. New Phytol 2005, 167(I): 165-170.

4I. Schubert R, Sperisen C, Muller-Starck G, La Scala S, Ernst D, Sanderman H, Hager KP: The cinnamyl alcohol dehydrogenase gene structure in Piicea abies (L.) Karst.: genomic sequences, Southern hybridization, genetic analysis and phylogenetic relationships. Trees 1998, 12:453-463.

42. Goffner D, Van Doorsselaere J, Yahiaoui N, Samaj J, Grima-Pettenati J, Boudet AM: A novel aromatic alcohol dehydrogenase in higher plants: molecular cloning and expression. Plant Mol Biol 1998, 36:755-765. 
43. Somssich IE, Wernert P, Kiedrowski S, Hahlbrock K: Arabidopsis thaliana defense-related protein ELI3 is an aromatic alcohol:NADP+ oxidoreductase. Proc Natl Acad Sci USA 1996, 93(24): $14 \mid 99-14203$

44. Simons L, Bultman TL, Sullivan TJ: Effects of methyl jasmonate and an endophytic fungus on plant resistance to insect herbivores. J Chem Ecol 2008, 34(I 2): I5 I I-I5I7.

45. Belhadj A, Saigne C, Telef N, Cluzet S, Bouscaut J, Corio-Costet MF, Merillon JM: Methyl jasmonate induces defense responses in grapevine and triggers protection against Erysiphe necator. J Agric Food Chem 2006, 54(24):9119-9125.

46. Ming R, Hou S, Feng $Y$, Yu Q, Dionne-Laporte A, Saw JH, Senin P, Wang W, Ly BV, Lewis KL, et al.: The draft genome of the transgenic tropical fruit tree papaya (Carica papaya Linnaeus). Nature 2008, 452(7 I 90):99I-996.

47. Velasco R, Zharkikh A, Troggio M, Cartwright DA, Cestaro A, Pruss D, Pindo M, Fitzgerald LM, Vezzulli S, Reid J, et al.: A high quality draft consensus sequence of the genome of a heterozygous grapevine variety. PLOS ONE 2007, 2(I 2):el 326.

48. Wingender E, Dietze P, Karas H, Knuppel R: TRANSFAC: a database on transcription factors and their DNA binding sites. Nucleic Acids Res 1996, 24(I):238-24I.

49. Lescot M, Dehais P, Thijs G, Marchal K, Moreau Y, Peer $Y$ Van de, Rouze P, Rombauts S: PlantCARE, a database of plant cis-acting regulatory elements and a portal to tools for in silico analysis of promoter sequences. Nucleic Acids Res 2002, 30(I):325-327.

50. Edgar RC: MUSCLE: a multiple sequence alignment method with reduced time and space complexity. BMC Bioinformatics 2004, 5: II3.

5I. Rodriguez FJ, Oliver JL, Marín A, Medina JR: The general stochastic model of nucleotide substitution. Journal of Theoretical Biology 1990, 142:485-50|.

52. Whelan S, Goldman N: A general empirical model of protein evolution derived from multiple protein families using a maximum-likelihood approach. Mol Biol Evol 200I, | 8(5):69|-699.

53. Guindon S, Gascuel O: A simple, fast, and accurate algorithm to estimate large phylogenies by maximum likelihood. Systematic Biology 2003, 52:696-704.

54. Chang S, Puryear J, Cairney J: A simple and efficient method for isolating RNA from pine trees. Plant Molecular Biology Reporter 1993, II:113-116.

Publish with Bio Med Central and every scientist can read your work free of charge

"BioMed Central will be the most significant development for disseminating the results of biomedical research in our lifetime. "

Sir Paul Nurse, Cancer Research UK

Your research papers will be:

- available free of charge to the entire biomedical community

- peer reviewed and published immediately upon acceptance

- cited in PubMed and archived on PubMed Central

- yours - you keep the copyright
BioMedcentral 